\title{
Rechtsgeschichte
}

www.rg.mpg.de

http://www.rg-rechtsgeschichte.de/rg13

Zitiervorschlag: Rechtsgeschichte Rg 13 (2008)

$\operatorname{Rg} 13200860-79$

http://dx.doi.org/10.12946/rg13/060-079

\section{Ruth Wolff}

Autorität und Authentizität: Zum Verhältnis von

Text und Siegel-Bild am Beispiel des Rechtsgutachtens Giovanni d'Andreas vom

9.5.1329 


\section{Abstract}

The analysis of the consilium of the famous canonist Giovanni d'Andrea and three of his Bolognese colleagues from 9th May 1329 reveals that legal consilia in the fourteenth century were usually authenticated both with the signature and signum of the notary drafting the document and with the seals of the jurists.

Three of the seal impressions formerly appended to the document illustrate in a strikingly similar fashion a detailed image widely disseminated on seals of jurists in medieval Italy: the doctor sitting enthroned in the cathedra, turned towards the viewer and at the same time concentrated on the open book in his hands.

The image is a sympathetic visualisation of the basic scheme of the consultation of the legal doctors, i. e. the transmission of the theoretical legal knowledge of the studium to the world outside the university, which is exemplified in the text of the document in a specific casus. Seals of medieval jurists bear an effigy of the owner of the seal, like other seals of high-ranking persons, such as emperors, kings and bishops. Thereby, and by the insistent repetition of a picture form which can be distinguished clearly by its three dimensionally refined visual idiom from the seals of emperors, kings and bishops, the seals of doctors of law advertise themselves as sigilla authentica which enjoy absolute credibility. 
* Der Beitrag stellt erste Ergebnisse des Projekts »Siegel-Bilder « vor, das unter der Leitung von Prof. Dr. Michael Stolleis (MPI für europäische Rechtgeschichte) und Prof. Dr. Gerhard Wolf (Kunsthistorisches Institut in Florenz - MPI) durchgeführt wurde.

I Florenz, Archivio di Stato, Diplomatico di S. Maria Nuova, 9.5.I329.

2 Aus der mittlerweile umfangreichen Literatur zu Rechtsgutachten seien hier zitiert: MARIo AsCHERI, Consilium sapientis, perizia medica e res judicata, diritto dei dottori e istituzioni comunali, in: Proceeding of the Fifth International Congress of Medieval Canon Law, Città del Vaticano I980, 533579; M. Ascheri, Saggio di bibliografia consiliare, in: DERS., Diritto medievale e moderno. Problemi del processo, della cultura e delle fonti giuridiche, Rimini

\section{Autorität und Authentizität: Zum Verhältnis von Text und Siegel-Bild am Beispiel des Rechtsgutachtens Giovanni d'Andreas vom 9.5.1329*}

Im Zentrum des vorliegenden Beitrags steht ein Rechtsgutachten vom 9. Mai I329, das der berühmte Rechtsgelehrte Gio-

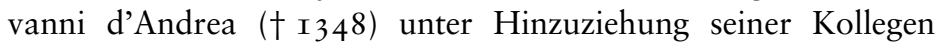
Giovanni Calderini, Filippo Formaglini und Azo de Raminghis verfasste. ${ }^{\text {I }}$ An das Gutachten waren ehemals die Abdrücke der Siegel der vier Juristen angehängt, die mittlerweile getrennt von dem Gutachten im Florentiner Staatsarchiv aufbewahrt werden. Im Folgenden soll der Zusammenhang von Text und Siegeln anhand dieses Gutachtens exemplarisch untersucht werden, wobei ein besonderes Verhältnis von Text und Siegel-Bild zu Tage tritt, das bislang weder von der rechtshistorischen noch von der kunsthistorischen Forschung in den Blick genommen wurde. Kernfragen dieses Verhältnisses sind diejenigen von Autorität und Authentizität, die sowohl die Bilder als auch die Texte, und nicht zuletzt ihr enges Beziehungsgeflecht, betreffen.

I. Typologie und diplomatisches Erscheinungsbild

der Rechtsgutachten in der bisherigen Forschung

Die Rechtsgeschichte hat sich in jüngerer Zeit verstärkt juristischen consilia zugewandt und eine Fülle von Materialien und Untersuchungsergebnissen zusammengetragen. ${ }^{2}$ Theorie und Praxis mittelalterlichen Ratgebens wurden darüber hinaus in einem breiteren kulturgeschichtlichen Rahmen in ihren theologischen Ursprüngen und Zusammenhängen, bezüglich juristischer und medizinischer Gutachten und consilia zur Magie untersucht. ${ }^{3}$ Die Geschichte juristischer Gutachten beginnt in der Mitte des I2. Jahrhunderts, seit der Mitte des I3. Jahrhunderts wachsen sie explosionsartig an und erleben im ausgehenden I3. und während des I4. Jahrhunderts ihren Triumph. ${ }^{4} \mathrm{M}$. Ascheri hat eine Typologie der Rechtsgutachten aufgestellt und unterscheidet vier

Mittelalter. Zum historischen Aussagewert einer Quellengattung, Sigmaringen I995 (Schriftenreihe des Deutschen Studienzentrums in Venedig, I3); Monica Chiantini, Il Consilium sapientis nel processo del secolo XIII. San Gimignano I 246-I3 I 2, Siena I994; Legal Consulting in the Civil Law Tradition, edited by M. AscherI, I. BAUMgÄrtner, J. Kirshner, Berkeley I999.
3 Consilium. Teorie e pratiche del consigliare nella cultura medievale, a cura di Carla Casagrande, Chiara Crisciani, Silvana VecCHIO, Firenze 2004 .

4 Mario Ascheri, I "consilia « dei giuristi: una fonte per il tardo Medioevo, in: Bullettino dell'Istituto storico italiano per il Medioevo CV (2003) 305-333, hier 3 Io. 
Typen, von denen für das vorliegende Thema nur zwei genannt werden sollen. ${ }^{5}$ Erstens das consilium sapientis, das vom Richter oder einer der beiden Parteien bzgl. zweifelhafter juristischer Punkte für die Urteilsfindung von sapientes, also gelehrten Juristen des allgemeinen Rechts, die außerhalb des Gerichtshofs tätig waren, erbeten wurde und für den Richter in seinem Urteilsspruch bindend war. Dieses war zumeist sehr knapp formuliert. Zweitens das consilium pro parte, mit dem eine oder mehrere der Parteien vor oder während des Prozesses gelehrte Juristen außerhalb des Gerichtshofs beauftragte, das aber für den Richter nicht bindend war. Die consilia pro parte sind im Gegensatz zu den consilia sapientis ausführlich formuliert, nicht zuletzt deshalb, weil sie den Richter überzeugen mussten. Ihre Ausführlichkeit machte sie anwendbar für andere, zukünftige Fälle, und sie wurden deshalb bereits um I 300 gesammelt und schließlich als Sammlungen gedruckt. ${ }^{6}$ Die consilia pro parte wurden ebenfalls vor Gericht präsentiert, gingen aber nicht wie die consilia sapientis in die Prozessakten ein. Allerdings wurden sie zumeist von den Kommunen oder kirchlichen Stellen, die sie in Auftrag gegeben hatten, im Original aufbewahrt und in die Libri iurium aufgenommen.

Ascheri hebt das feierliche Erscheinungsbild der consilia pro parte hervor: Oft seien sie auf ganzen Pergamentbögen geschrieben und in den meisten Fällen mit dem Siegel des Konsulenten besiegelt. Im I3. und bis zum Ende des I4. Jahrhunderts seien sie von Notaren ausgestellt, die Inhalt und Urheberschaft des consiliums authentisierten. Erst gegen Ende des I4. Jahrhunderts seien Rechtsgutachten auf Papier geschrieben und ohne die Redaktion eines Notars mit der eigenhändigen Unterschrift des Konsulenten und seinem jetzt direkt in das Papier eingedrückten und nicht mehr anhängenden Siegel authentisiert. ${ }^{7}$

Die Siegel selbst und ihre Bilder haben in der bisherigen Forschung noch kein Interesse gefunden. Die eher raren Abbildungen von Urkunden zeigen so zumeist auch nicht die anhängenden Siegel, oder wenn doch, in einer Bildqualität, die weder die Siegelumschrift noch das Siegelbild erkennen lässt.

5 AsCHERI, I "consilia « dei giuristi 3I4-3 I9.

6 Die ersten Sammlungen von Consilia sind diejenigen von Dino del Mugello († I303?), sodann von Oldrado da Ponte († kurz nach I337) und Federico Petrucci ( nach I343), s. Vincenzo Colli, Consilia dei giuristi medievali e produzione libraria, in: Legal Consulting, I73-225.
7 Mario Ascheri, I consilia come acta processuali, in: La diplomatica dei documenti giudiziari. Dai placiti agli acta - secc. XII-XV, Bologna, I 2-I 5 sett. 200I, a cura di Giovanni Nicolaj, Roma 2004, 309-328, hier 32I f. 
2. Ähnlichkeiten - das diplomatische Erscheinungsbild des

Rechtsgutachtens und seiner Siegel

Giovanni d'Andreas Rechtsgutachten vom 9. Mai I329 handelt von dem Patronatsrecht der Familie der Portinari am Hospital und der Kirche von Santa Maria Nuova in Florenz, und

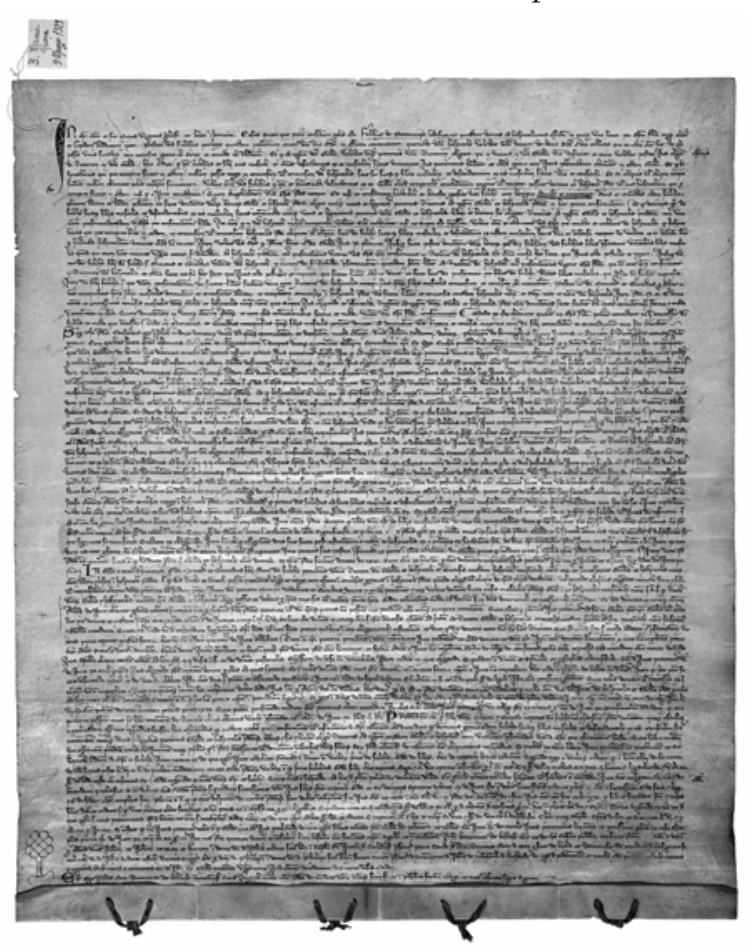

Bild I: Rechtsgutachten des Giovanni d'Andrea vom 9.5.I329 (Florenz, Archivio di Stato, Diplomatico di S. Maria Nuova, 9.5.1329), Urkunde A genauer von dem Recht, den Präfekten des Hospitals zu präsentieren. Das berühmte Florentiner Hospital, das eines der ersten werden sollte, bei dem die medizinische Versorgung im Vordergrund stand, war I 288 von Folco Portinari, dem Vater von Dantes Beatrice, gegründet worden, der in seinem Testament bestimmt hatte, dass das Patronat »in perpetuum « seinen männlichen Nachkommen zukomme. ${ }^{8}$ Giovanni d'Andreas consilium hatte einen Schiedsspruch des Dominikaners Uberto di Guido am 3.8.1329 zur Folge, der den männlichen Portinari das alleinige Recht der Auswahl des Vorstehers des Hospitals zuerkannte. ${ }^{9}$ Das Gutachten ist ein consilium pro parte, das von den Söhnen Fulco Portinaris, Maneto und Gerado, in Auftrag gegeben wurde. Ascheri, der das Gutachten publiziert, vermerkt nicht, dass unter dem Datum des 9. Mai I 329 zwei besiegelte Pergamenturkunden im Florentiner Staatsarchiv aufbewahrt werden. ${ }^{\text {IO }}$ (Bild I und Bild 2) Beide Urkunden zeigen das von Ascheri beschriebene feierliche Erscheinungsbild und sind jeweils auf einem großen Pergamentbogen von dem Notar Giovanni del fu Benvenuto da Belviso geschrieben und mit seinem Notariatszeichen versehen, und beide waren ursprünglich mit vier anhängenden Siegeln versehen, von denen sich an den Urkunden nur noch die Schnüre erhalten haben. Die vier Rechtsgelehrten bestätigen in beiden Exemplaren jeweils am Ende des Gutachtens, dass sie die Siegel anbringen ließen und außerdem den Notar beauftragten, aus dem consilium ein öffentliches Instrumentum zu machen. ${ }^{\text {II }}$ Der Notar bestätigt seinerseits in der Subskriptionszeile, dass er den vorangegangen Text im Auftrag der

8 Die Gründung des Hospitals erfolgte am 23.6.I288; Fulcos Testament wurde bereits am I 5.I. I 288 ausgestellt. Zu Fulco Portinari s. ARnaldo D'Addario, Art. $»$ Portinari, Fulco «, in: Enciclopedia Dantesca, Bd. IV, Roma I973, 608. Zum Hospital s. Il R. Arcispedale di S. Maria Nuova: I suoi benefattori, sue antiche memorie, Firenze I 888 und La bellezza come terapia. Arte e assistenza

vegno Internazionale, Firenze 2022 maggio 2004, a cura di ENRICO Ghidetti e Esther Diana, Firenze 2005.

9 Vgl. L. Passerini, Storia degli stabilimenti di beneficenza e d'istruzione elementare gartuita della città di Firenze, Firenze I 853 , 29I.

Io Zunächst in: MARIo AsCheri, Analecta manoscritta consiliare (I285-I354), in: Bulletin of the medieval canon law I 5 (I985) 6I94, hier IV, 86-92, sodann ein zweites Mal in: Maria Ascheri, I " consilia « dei giuristi: una fonte per il tardo Medioevo, in: Bullettino dell'Istituto storico italiano per il Medioevo CV (2003) 305333 , hier Nr. I, 328-330.

I I facientes apponi sigilla ac etiam mandantes (...) notario ut de ipso consilio publicum conficiat instrumentum. 
besagten Rechtsgelehrten geschrieben, in » Öffentlicher Form « redigiert und mit seinem gewohnten Zeichen signiert habe. ${ }^{\mathbf{I 2}}$ Offensichtlich hat sich der Notar bemüht, beiden Exemplaren des Gutachtens das gleiche Erscheinungsbild zu geben, was die Größe des verwendeten Pergamentbogens angeht, die Zeilenzahl, aber auch hinsichtlich der Zierinitiale und der den Text gliedernden Großbuchstaben oder der Hervorhebung des $\mathrm{Na}$ mens des ursprünglichen Patrons des Hospitals von Santa Maria Nuova, Fulco Portinari, jeweils etwa in der Mitte der ersten Zeile der Urkunden. Erst bei genauerem Hinsehen zeigen sich minimale Unterschiede der beiden Urkundenexemplare: Bei der Zierinitiale der Urkunde A ist so z. B. der obere Balken über dem I ein wenig über den Stamm des I fortgesetzt, während dieser Balken in der Urkunde B über dem Stamm des I endet. Die nach unten offenen Rundungen des Notariatszeichen der Urkunde A sind ein wenig größer als diejenigen auf der Urkunde B. Die Urkunde A hat 77, die Urkunde B nur 76 Zeilen. Sowohl bei der Urkunde A als auch bei der Urkunde B sind zudem am linken und rechten Textrand Worte ergänzt, die im Haupttext vergessen wurden. ${ }^{\mathbf{I} 3}$ Der Text beider Urkunden stimmt jedoch Wort für Wort miteinander überein.

Im Inventar der »sigilli staccati « des Florentiner Staatsarchivs, also der Sammlung von ca. 800 Siegelabdrücken, die von ihren Urkunden getrennt aufbewahrt werden, sind unter dem Datum des 9. Mai I329 tatsächlich insgesamt acht Siegelabdrücke aufgeführt, d. h. jeweils zwei Abdrücke des Siegels Gio-

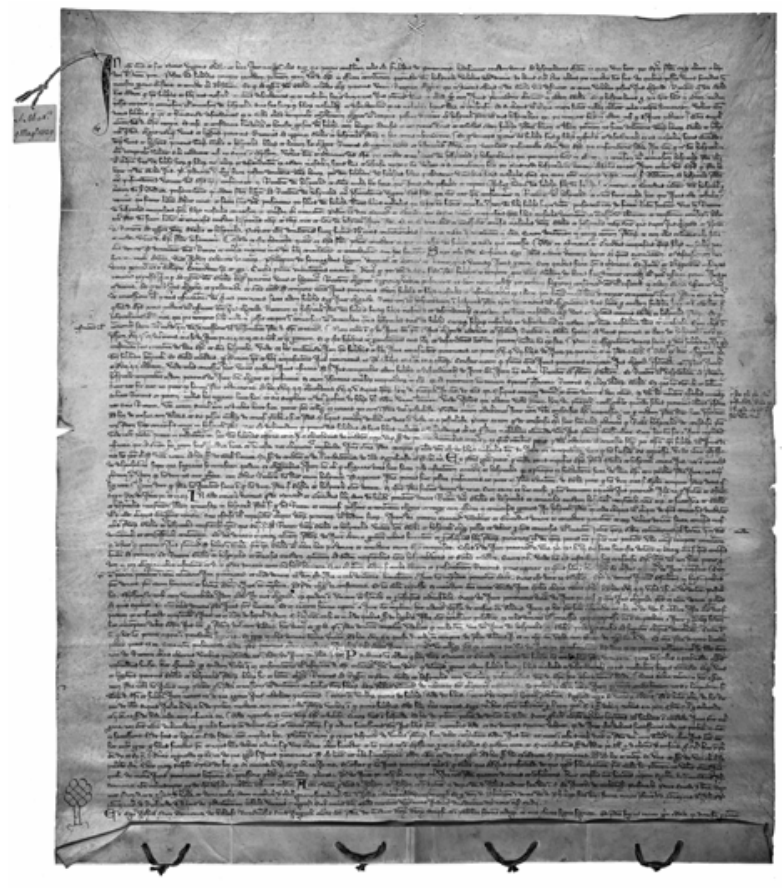

Bild 2: Rechtsgutachten des Giovanni d'Andrea vom 9.5.I329 (Florenz, Archivio di Stato, Diplomatico di S. Maria Nuova, 9.5.1329), Urkunde B vanni d'Andreas, Giovanni Calderinis, Filippo Formaglinis und Azo de Raminghis'. ${ }^{\mathbf{4}}$ Das Siegel Azo de Raminighis', das kleiner als die anderen und nicht spitzoval, sondern rund ist, ${ }^{\mathbf{1 5}}$ hat sich in beiden Exemplaren nur schlecht konserviert. Sein Siegelbild ist kaum zu erkennen, möglicherweise handelt es sich um ein Tier. Einer der beiden Siegelabdrücke Giovanni d'Andreas ist zudem nur fragmentarisch erhalten. Es ist jedoch deutlich zu sehen, dass

I2 Et ego Johannes condam Benuennuti de Beluiso Bononiensis ciuis imperiali auctoritate notarius predicta de mandato dictorum dominorum scripsi et in publicam formam redegi a meo consueto signo signaui.

I3 Die Tatsache, dass die Urkunde B an vier Stellen Textergänzungen am Rand vorweist, während der Text der Urkunde B nur an zwei Stellen und mit jeweils nur einem
Wort am Rand ergänzt ist, ließe allenfalls darauf schließen, dass der Notar zuerst die Urkunde B geschrieben hat. Und doch ist nicht eine Urkunde die Kopie der anderen, denn bei beiden Urkunden handelt es sich um selbständige Schriftstücke, die nicht aufeinander verweisen, sondern in voller Unabhängigkeit voneinander bestehen können und jeweils mit vier Siegeln versehen sind.
I4 Der Archivar P. Berti führte Ende des I9. Jahrhunderts von Urkunden abgetrennte Siegelabdrücke aus verschiedenen kleineren Sammlungen zusammen und trennte weitere Siegel von Dokumenten $\mathrm{ab}$. In seinem handschriftlichen Inventar ordnete er das Material in fünf Reihen und in jeweils chronologischer Folge an. Unter dem Datum des 9.5.I329 sind jeweils zwei Abrücke des Siegels von Giovanni d'Andrea (Nr. 82 und Nr. 83, wobei Nr. 82 nur noch in einem Fragment erhalten ist), von Giovanni Calderini (Nr. 84 und Nr. 85), von Filippo Formaglini (Nr. 86 und Nr. 87) und von Azo de Raminghis (Nr. 88 und Nr. 89) aufgeführt.

I 5 Die Siegelabdruck Azo de Raminghis' misst nur etwa $2 \mathrm{~cm}$ im Durchmesser. 
I6 Robert Davidsohn, Geschichte von Florenz, Vierter Band: Die Frühzeit der Florentiner Kultur, Zweiter Teil: Gewerbe, Zünfte, Welthandel und Bankwesen, Berlin 1925 , hier 123 .

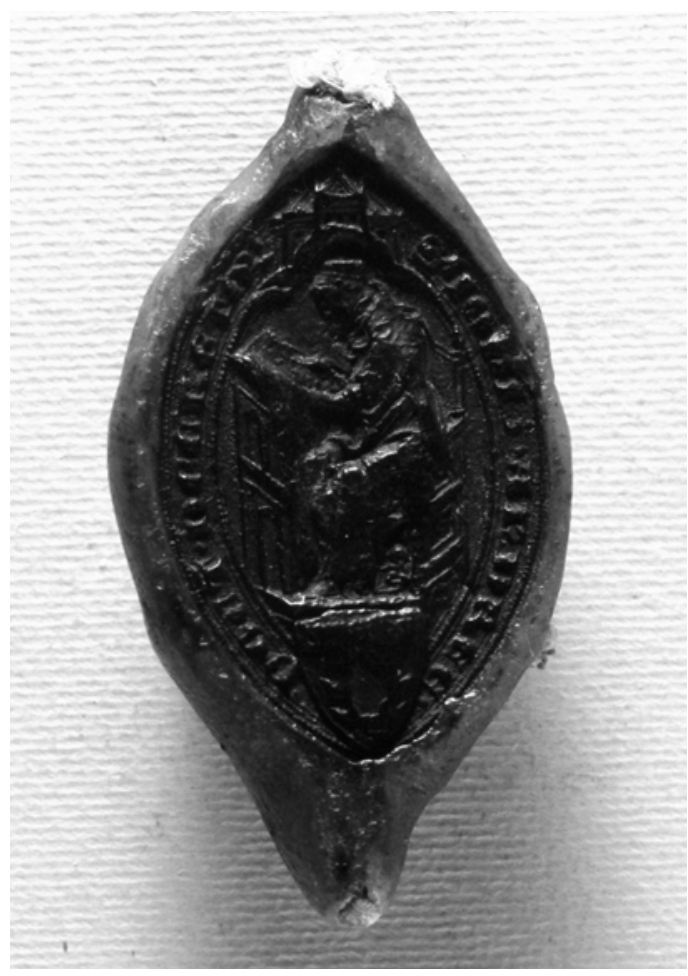

Bild 3: Abdruck des Siegels von Giovanni d'Andrea vom œ $\quad 9.5 .1329$ (Florenz, Archivio di Stato, »sigilli staccati«, ก Nr. 83) jeweils ein Siegelstempel zwei Abdrücke erzeugt hat, bei denen Wachs der gleichen Farbe verwendet wurde. Der Stempel Giovanni d'Andreas wurde zweimal in fast schwarzes Wachs abgedrückt, während die Stempel Giovanni Calderinis, Filippo Formaglinis und Azo de Raminghis' jeweils zweimal in rotes Wachs eingedrückt wurden. Die sechs Siegelabdrücke sind in eine schützende Schale aus naturfarbenem Wachs eingebettet.

Abgesehen von der unterschiedlichen Farbe des Wachses, durch die vor allem die Abdrücke des Siegels von Giovanni d'Andrea hervorstechen, sind sich die Siegelabdrücke Giovanni d'Andreas, Filippo Formaglinis und Giovanni Calderinis sehr ähnlich, denn sie zeigen im spitzovalen Format jeweils das gleiche Motiv, den Rechtsgelehrten, der in seiner Kathedra vor einem Pult thront und ein geöffnetes Buch in den Händen hält (Bild 3, 4 und 5).

\section{Das Bildmotiv in der bisherigen Siegelforschung}

Das Gutachten Giovanni d'Andreas vom 9. Mai I329 veranschaulicht auf plastische Weise die Dominanz des Bildmotivs des doctor in cathedra auf Siegelabdrücken an Rechtsgutachten, auf die bereits Robert Davidsohn in seiner Geschichte von Florenz aus dem Jahr I925 aufmerksam machte. ${ }^{\mathbf{1 6}}$ Davidsohns Hinweis blieb in der Siegelkunde unbeachtet, unter anderem deshalb, weil die

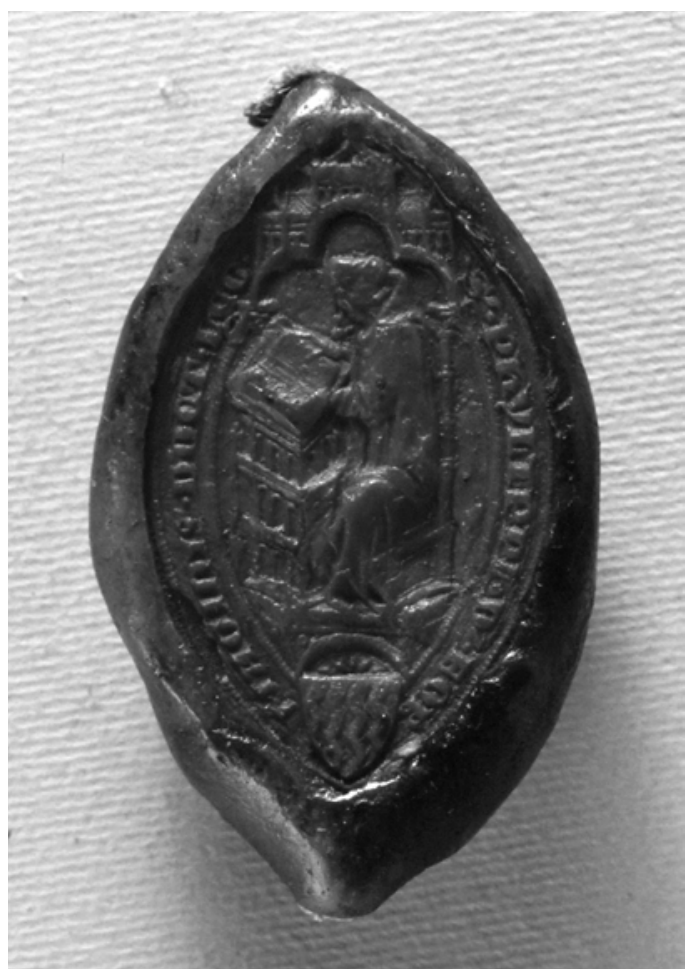

Bild 4: Abdruck des Siegels von Filippo Formaglini vom 9.5.I329 (Florenz, Archivio di Stato, »sigilli staccati«, Nr. 86) 
internationale Siegelforschung seit ihren Anfängen und bis heute entscheidend von Frankreich bestimmt wird. In Frankreich wurden bereits im I9. Jahrhundert große Siegel-Repertorien von Siegelabdrücken anlegt, von denen jeweils ein Abguss konserviert wurde. ${ }^{\mathbf{I 7}}$ In jüngerer Zeit manifestiert sich die Vitalität der französischen Siegelforschung u. a. in der Erstellung des Corpus des sceaux français du moyen age. ${ }^{18}$ Das umfangreiche erfasste Material ist in Frankreich, aber auch in anderen Ländern nach französischem Vorbild nach Siegelführern geordnet. Rechtsgelehrte aber werden unter den Siegelführern nicht aufgeführt. Die Kritik auch der französischen Siegelforschung an der Klassifizierung nach Siegelführern führte u. a. dazu, dass auch die in der deutschen Siegelkunde vorherrschende Klassifizierung nach Bildtypen Eingang in die internationale Siegelforschung fand, die mit dem Vocabulaire internationale de la sigillographie eine Bildtypologie von Siegeln vorschlägt. ${ }^{\text {I9 }}$ Das Bild des in der Kathedra thronenden Gelehrten ist in dieser Bildtypologie nicht vertreten. ${ }^{20}$

In Italien bedeutet Siegelforschung dagegen in erster Linie die Untersuchung von Siegelstempeln, die sich in großer Anzahl erhalten haben und in den beiden größten Sammlungen des Florentiner Nationalmuseums, dem Bargello, und im Palazzo Venezia in Rom wie auch in vielen kleineren Museen zusammengeführt sind. Die immer noch grundlegende Untersuchung zu italienischen

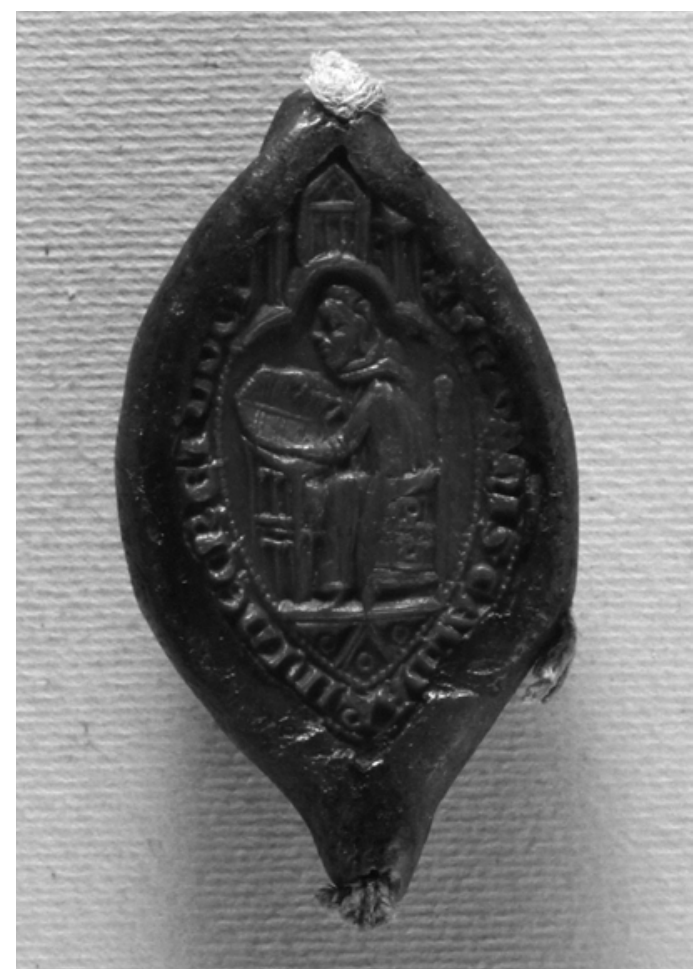

Bild 5: Abdruck des Siegels von Giovanni Calderini vom 9.5.I329 (Florenz, Archivio di Stato, »sigilli staccati«, Nr. 84)
I7 G. Demay, Inventaire des sceaux de l'Artois et de la Picardie recueillis dans les dépôts d'Archives, musées et collections particulières des départements du Pas-de-Calais, de l'Oise, de la Somme et de l'Aisne ..., avec un catalogue de pierres gravées ayant servi à sceller, Paris I 877; DERs., Inventaire des sceaux de la collection Clairambault à la Bibliothèque nationale, Paris I 877; DERs., Inventaire des sceaux de la Flandre, recueillis dans les dépôts d'Archives, musées et collections particuliéres du département du Nord, 2 Bde., Paris I873; DERS., Inventaire des sceaux de la Normandie, avec une introduction sur la paléographie des sceaux, Paris I88 I; L.-C. DouËT D'Arq, Collection de sceaux, 3 Bde., Paris I863-I868 (I872).

I 8 Bislang sind erschienen: Corpus des sceaux français du Moyen Age, Bd. I: Les sceaux des villes, par B. Bedos, Paris, I980 und Corpus des sceaux français du Moyen Age, Bd. II: Les sceaux des rois et de régence, par M. DALAs, Paris I99I.

I9 Die deutsche Siegelkunde des I9. Jahrhunderts ging mit F. L. vON HOHENLOHE-WALDENBURG, Sphragistische Aphorismen. 300 mittelalterliche Siegel systematisch klassifiziert und erläutert, Walluf (bei Wiesbaden) I973, unver. Nachdruck der Ausgabe von I 882, bei der Klassifizierung der Siegel allein davon aus, was das Siegel selbst visualisiert und unterschied vier große Kategorien: Schriftsiegel, Bildsiegel, Porträtsiegel und Wappensiegel. Diese grobe Einteilung wurde von ToNI DiEDERICH, Prolegomena zu einer neuen Siegeltypologie, in: Archiv für Diplomatik 29 (I983) 242248 zu 24 Bildttypen erweitert, die er 28 Siegelführergruppen gegenüberstellt. Gelehrte sind nicht bei den Siegelführergruppen aufgeführt, ebenso wenig wie das Bild des Doctor in cathedra bei den Bildtypen.

20 Vocabulaire International de la Sigillographie, hg. v. Conseil International des Archives. Comité de sigillographie (Ministero per i beni culturali e ambientali Pubblicazioni degli archivi di Stato Sussidi 3), Roma I990. 
2I G. C. BASCAPÉ, Sigillografia. Il sigillo nella diplomatica, nel diritto, nella storia, nell'arte, 3 Bde., Milano I969-I984.

22 Rom, Museo di Palazzo Venezia, Sammlung Tagliavini, Inv. Nr. 7. Vgl. Elisabetta Cioni, Il sigillo a Siena nel medioevo, Siena Palazzo Pubblico, 25.2.-I9.3.I989, Siena I989, Kat. Nr. I8. Der Siegelstempel ist aus Bronze, rund und misst 4I mm im Durchmesser. Die Siegelumschrift lautet: + Sigillum BLSII.LEGVm.DOCTOrIS DE MONTANINIs.De.SENis.

23 Siena, Museo Civico, Inv. Nr. 20I. Vgl. Cioni, Il sigillo a Siena nel medioevo, Kat. Nr. I7. Der Siegelstempel hat einen Durchmesser von $28 \mathrm{~mm}$, die Umschrift lautet: LANDI.IVDICIS. DomiNI.BARTALOMeI.DE STELLIS.

$\stackrel{2}{\circ} \quad 24 \begin{aligned} & \text { BASCAPÉ, Sigillografia, Bd. I: Si- } \\ & \text { gillografia generale. I sigilli pub- }\end{aligned}$

Siegeln, Giacomo Bascapès Sigillografia, ${ }^{2 \mathbf{I}}$ basiert ebenfalls vor allem auf Siegelstempeln. Die hier in unterschiedlichen Zusammenhängen erwähnten Beispiele für die Siegel von Rechtsgelehrten zeigen, dass die meisten von ihnen das Motiv des Doctor in Kathedra veranschaulichen. Als Beispiel sei hier das Siegel des Biagio Montanini, doctor legum aus Siena, genannt, das sich im Museum des Palazzo Venezia in Rom befindet und auf den Beginn des I4. Jahrhunderts datiert wird. ${ }^{22}$ Wie die Rechtsdoktoren Giovanni d'Andrea, Giovanni Calderini und Filippo Formaglini thront auch ihr Sieneser Kollege in seiner Kathedra vor einem Pult, auf dem ein geöffnetes Buch liegt, dem er sich zuwendet. Sein Siegel ist rund, und das (beschädigte) Familienwappen vor dem Pult eingeblendet. Aber nicht nur Doktoren des Rechts, sondern zum Beispiel auch Richter führten Siegelstempel mit dem Motiv, wie Lando delle Stelle, dessen bronzener Siegelstempel vom Ende des I 3. Jahrhunderts im Museo Civico in Siena konserviert wird (Bild 6). ${ }^{23}$ Auch einige Mediziner wählten das Motiv des Doctor in Cathedra für ihre Siegelstempel, ${ }^{24}$ wie z. B Leuzio, doctor logice et medicinalis scientiae, dessen Siegel in die zweite Hälfte des I 3 . Jahrhunderts datiert wird. ${ }^{25}$

\section{Das Bildformular}

Die Siegelabdrücke Giovanni d'Andreas, Giovanni Calderinis und Filippo Formaglinis veranschaulichen also ein Motiv, das im Italien des Mittelalters auf Siegeln von Rechtsgelehrten weit verbreitet war. Die Ähnlichkeit der Siegelabdrücke der drei Rechtsdoktoren beschränkt sich allerdings nicht auf das Motiv, sondern umschließt auch die Art und Weise, in der es in Szene gesetzt wird. So ist der in der Kathedra thronende Rechtsgelehrte immer in der gleichen Haltung visualisiert. Die Kathedra ist immer auf der vom Betrachter aus rechten Bildhälfte abgebildet und das Pult auf der linken. Das Buch liegt nicht völlig geöffnet auf dem Pult, sondern wird immer vom Gelehrten zu Dreivierteln geöffnet mit seinen beiden Händen gehalten. Der Kopf des Gelehrten ist bei allen Siegeln dem Buch zugeneigt. Gelehrter, Kathedra und Pult füllen jeweils das Siegelbild zum Großteil und vor einer ansonsten von anderen Bildmotiven oder Dekorationen leer gelassenen Bildfläche aus. Die Bildfläche wird von der Siegelumschrift gerahmt, die durch feine Perlleisten vom Bildfeld abgetrennt ist. Im oberen

blici e quelli privati, 9I: $»(\ldots)$ quelli (sigilli, Erg. v. mir) di teologi, dottori di diritto, canonisti, notai o giudici ecclesiastici, li ritraggono seduti presso il leggìo o lo scrittoio, nell'atto di leggere, di insegnare, di sentenziare. (...) Magistrati laici, notai, giudici, appaiono seduti in cattedra o allo scrittoio, come quelli ecclesiastici, e cosi pure i maestri e qualche medico."
25 Florenz, Museo Nazionale del Bargello, Inv. Nr. 935, Sigilli nel Museo Nazionale del Bargello, a cura di ANdrea Muzzi, Bruna Tomasello, Attilio Tori, Bd. III: Sigilli di enti civili, Firenze I990, Kat. Nr. 354, I 54. Der runde bronzene Siegelstempel misst $33 \mathrm{~mm}$ und trägt die Siegelumschrift: + S.'LEUTII. DOCT. LOGICE. ET. MEDICINAL'. SCI $\overline{\mathrm{E}}$. 
Abschluss des Siegelovals ist das Bildfeld jeweils von einem Dreipass gerahmt, der von einer Architektur mit einer begiebelten Tempelfront in der Mitte bekrönt wird. Der im Profil abgebildete Kopf des Gelehrten ist unter dem zentralen und höchsten Rundbogen des Dreipasses mit der Tempelfront darüber platziert. Der untere Abschluss des Siegelovals wird bei den Siegeln Giovanni d'Andreas und Filippo Formaglinis von ihrem Familienwappen ausgefüllt, das die Siegelumschrift überdeckt und auf der das Bild des in der Kathedra thronenden Gelehrten ruht. Der entsprechende Bildraum auf dem Siegel Giovanni Calderinis ist von einer Dekoration mit Dreiecken und Kreisen ausgefüllt, möglicherweise weil seine Familie kein Wappen führte. Das Pult auf den Siegeln aller drei Rechtsgelehrter ist jeweils mit schmalen, runden Bögen dekoriert, bei Giovanni Calderini in zwei, bei den anderen Rechtsgelehrten in drei übereinander liegenden Zonen. Die Kathedra hat bei allen drei Siegeln eine hohe Rückenlehne, die Seitenlehne ist verziert und der Mantel des Rechtsgelehrten hängt ein wenig über die Seitenlehne.

Die Siegelabdrücke der drei Rechtsgelehrten zeigen also ein sorgfältig konstruiertes Bildformular, das jeweils in allen Details auf kleinster Bildfläche wiederholt wird, auch wenn deutlich künstlerische Unterschiede erkennbar sind. Auch die oben erwähnten Siegelstempel des Richters Lando delle Stelle und des Rechtsdoktors Biagio Montanini visualisieren dieses Bildformular in wichti-

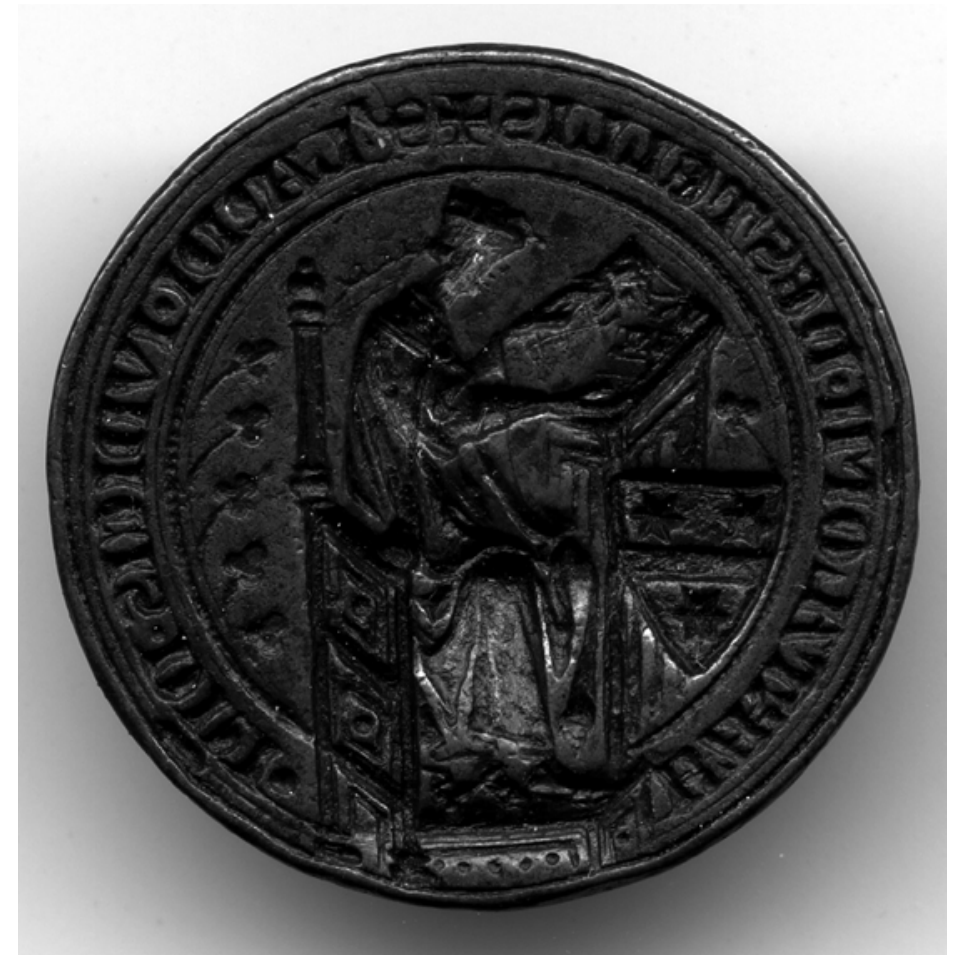

Bild 6: Siegelstempel des Richters Lando delle Stelle (Siena, Museo Civico, Inv. Nr. 20I) 
gen Grundzügen: Der Richter und der Rechtsdoktor thronen ganzfigurig und zentral in der rechts gezeigten Kathedra vor dem Pult links, sie halten das Buch zu Dreivierteln geöffnet in ihren Händen und wenden sich ihm zu. Allerdings wird hier auf die Präsentation des Gelehrten unter dem von einer Tempelfront bekrönten Dreipass verzichtet und die Wappen sind seitlich und nicht unterhalb der Bildfigur platziert, wohl vor allem aufgrund des runden und nicht spitzovalen Formats dieser Siegelstempel.

In wichtigen Grundzügen ist das Bildformular, mit dem das Motiv des Doctor in Cathedra auf den Siegelabdrücken Giovanni d'Andreas, Giovanni Calderinis und Filippo Formaglinis präsentiert wird, also bereits zu Beginn des I4. Jahrhunderts in Italien ortsübergreifend verbreitet. Das bezeugt auch ein Siegelstempel Giovanni d'Andreas, der heute im Museo Civico Medievale in Bologna aufbewahrt wird und der ein anderer sein muss als derjenige, der die Siegelabdrücke von I329 produziert hat (Bild 7). Dieser Siegelstempel wurde im Jahr 2006 in der Ausstellung Giotto e le arti a Bologna al tempo di Bertrando del Poggetto präsentiert. ${ }^{26}$ Er ist spitzoval und die Siegelumschrift lautet wie die der Abdrücke aus dem Jahr I 329: S(igillum) Ioh(ann)is Andree Doct(oris) Decretor(um). Aus stilistischen Gründen wird der Stempel im Katalogbeitrag auf das Ende des I3. bzw. den Anfang des I4. Jahrhunderts

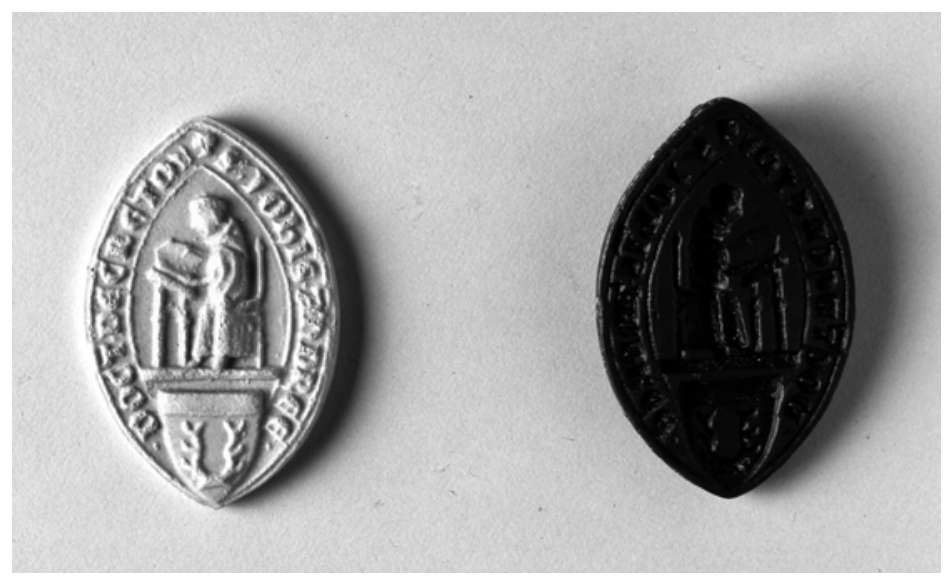

Bild 7: Siegelstempel des Giovanni d'Andrea (Bologna, Museo Civico Medievale, Inv. Nr. 3929) und moderner Abguss dieses Stempels

26 Bologna, Museo Civico Medievale, Inv. Nr. 3929; Giotto e le arti a Bologna al tempo di Bertrando del Poggetto, hg. v. Massimo MediCA, Milano 2005, Katalogbeitrag von Silvia Giorgi, II 2 und Abb. auf II3. 
datiert, wobei seine frühen gotischen Formen hervorgehoben werden. ${ }^{27}$ Auch die Abdrücke des Siegels von Giovanni d'Andrea im Florentiner Staatsarchiv werden im entsprechenden Katalogbeitrag erwähnt, wobei allerdings nicht bemerkt wird, dass sie keinesfalls von dem in der Ausstellung präsentierten Stempel stammen können. Der Stempel ist wohl tatsächlich in die Anfangszeit der universitären Karriere Giovanni d'Andreas zu datieren, kann aber nicht vor I298, dem Jahr seiner Promotion zum doctor decretorum geschaffen worden sein, da die Siegelumschrift ihn als solchen ausweist. ${ }^{28}$ Von dem nicht erhaltenen Siegelstempel, der den Abdruck am Gutachten von 1329 erzeugte, hat sich hingegen ein weiterer Abdruck, diesmal nicht aus fast schwarzem, sondern aus rotem Wachs im Staatsarchiv von Florenz erhalten, der an eine Urkunde vom 30. Mai I 3 I 5 angehängt war (Bild 8). ${ }^{29}$ Wieder authentisierte das Siegel ein Rechtsgutachten Giovannis, hier für die Bauhütte des Florentiner Baptisteriums, mit dem es gelang, die Opera vor der Auferlegung des päpstlichen Zehnts zu bewahren. ${ }^{3 \circ}$

Den im Bologneser Museum konservierten Siegelstempel Giovanni d'Andreas und den nicht erhaltenen Siegelstempel des Rechtsdoktoren, der die Abdrücke von I3 I 5 und I 329 im Florentiner Staatsarchiv erzeugt haben muss, trennen also im Höchstfall I7 Jahre. Beide zeigen das gleiche Bildformular, an dem der berühmte Bologneser Kanonist festhielt, auch wenn es alles andere als neu und nicht auf Siegel von Rechtsgelehrten der bedeutenden Universitätsstadt Bologna beschränkt war. Der Vergleich des Siegelstempels mit den Siegelabdrücken verdeutlicht jedoch, worauf es dem Rechtsgelehrten bei seinem späteren, nicht mehr erhaltenen Siegelstempel angekommen sein muss.

Auf dem Siegelstempel aus dem Museo Civico in Bologna beansprucht das Wappen weitaus mehr Bildraum als auf den Abdrücken des Florentiner Staatsarchivs, auf denen die Figur des Rechtsgelehrten im Vergleich größer veranschaulicht werden kann. Das Bildfeld, in dem das

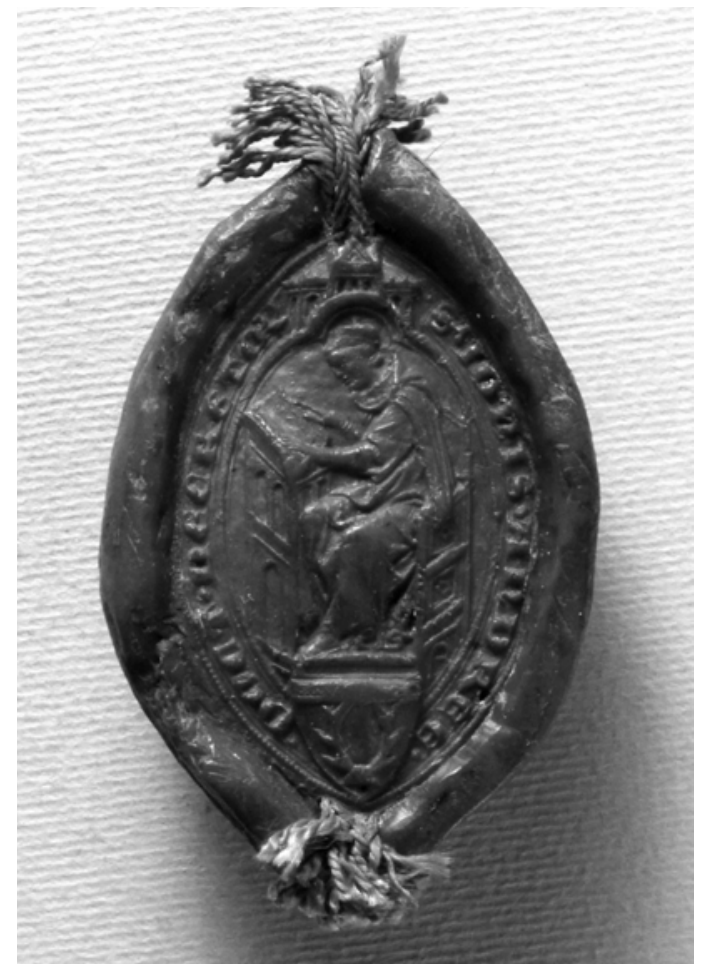

Bild 8: Abdruck des Siegels von Giovanni d'Andrea vom 30.5.I3 I 5 (Florenz, Archivio di Stato, » sigilli staccati«, Nr. 62)
27 Giotto e le arti a Bologna, I I 2 mit Verweis auf Renzo GRANDI, I monumenti dei dottori e la scultura a Bologna (I 267-I348), Bologna I982, I66: "Il sigillo, conservato al Museo civico, resta testimonianza importante, ad una data presumibilmente molto precoce, di orientamenti già in tutto gotici.«

$28 \mathrm{Zu}$ Giovanni d'Andreas Werdegang und Promotion s. Giorgio
Taмba, Art. » Giovanni d'Andrea «, in: Dizionario Biografico degli Italiani, Bd. 55, 2000, 667672, hier bes. 668 .

29 Staatsarchiv von Florenz, Diplomatico Mercatanti, 30.5.I3I 5. Der Siegelabdruck ist im Inventar der »sigilli staccati « des Staatsarchivs von Florenz unter der Nr. 62 verzeichnet.

30 Auf das Gutachten wies bereits Robert Davidsohn, Storia di
Firenze, Bd. IV, Firenze 1965, 248 mit Anm. 2, hin, datierte es aber irrtümlich auf das Jahr I306. Es wurde zum ersten Mal in Lapus de Castiglionchio, Allegationes, nu. I40, Venetiae I 57I, fol. I 7 orb-vb ediert. Vgl. AsCHERI, Analecta consiliare, Nr. 8, 66. 
3I Zur juristischen "lectio « als laut vorgetragener s. M. BELLOMO, Saggio sull'Università del diritto comune, Catania I979, 78-79. Zum mündlichen Charakter des Universitätsunterrichts J. MIETHKE, Die mittelalterlichen Universitäten und das gesprochene Wort, in: Historische Zeitschrift CCLI (I990) I-44; bei den studia der Bettelorden: G. Severino Polica, Libro, lettura, »lezione " negli Studia degli ordini mendicanti (sec. XIII), in: Le scuole degli ordini mendicanti (sec. XIII-XIV), Todi 1978, 305-37I, 373-4I3; allgemein: Luoghi e metodi d'insegnamento nell'Italia medievale (sec. XII-XIV), a cura di L. GARGan e O. Limone, Lecce, Galatina I989.

$32 \mathrm{Zu}$ den Gelehrtengrabmälern Bolognas s. u. a. GRANDI (Fn. 27), zu denjenigen in Padua Jill Emilee CARrington, Sculpted Tombs of the Professors of the University of N Padua, c. I358-c.I557, Ann Ar-

Wappen dargestellt ist, ist auf dem Stempel durch eine einfache horizontale Leiste von dem darüber liegenden mit dem Rechtsgelehrten separiert, auf den Abdrücken dagegen ist der obere horizontale Abschluss des Wappens selbst die Basis für den Aufbau von Kathedra und Pult, die nicht mehr in distanzierter Seitenansicht, sondern in umgekehrter perspektivischer Verkürzung zum Betrachter hin geöffnet dargestellt sind und die wir uns jetzt in ihrem Zusammenhang dreidimensional als Polyeder auf hexagonalem Grundriss vorstellen können. Der Gelehrte, dessen Beine nun in eleganter Überkreuzung zu sehen sind, ist in seiner Sitzhaltung auf den Betrachter hin ausgerichtet, auch wenn sich der Oberkörper dem Pult mit dem aufgeschlagenen Buch zuwendet und der Kopf im Profil dem Buch zugeneigt ist. Mit anderen Worten: Die Figur ist in Bewegung geraten, sie öffnet sich einerseits zum Betrachter hin, während andererseits die Beziehung des Gelehrten zum Buch intensiviert ist. Der Siegelstempel zeigt zudem noch nicht den rahmenden Dreipass mit der bekrönenden Architektur. Seine Hinzufügung auf den Siegelabdrücken trägt zur größeren Plastizität des Bildeindrucks bei und nobilitiert darüber hinaus den Gelehrten, der wie unter einem Baldachin in seiner Kathedra thront.

Dasjenige, was auf dem frühen Siegelstempel aus dem Bologneser Museum hölzern und starr ausgedrückt ist, wird auf den späteren Siegelabdrücken im Florentiner Staatsarchiv demnach gesteigert und pointiert formuliert, wie die Zentralität der Bildfigur des Rechtsgelehrten, sein autoritatives Gewicht und seine Konzentration auf das Buch, aber auch seine Öffnung nach außen, die auf dem Siegelstempel bereits durch das vom Gelehrten geöffnete Buch ausgedrückt ist. Denn die Hinwendung zum Buch, das die Hände geöffnet halten, meint nicht das stumme Lesen und Studieren, sondern lautes Lesen, also Vorlesen und Erklären (praelegere), ${ }^{3 \mathbf{I}}$ wie es vor allem im Unterricht an der Universität geschieht und auf zahlreichen Grabmälern von Rechtsgelehrten besonders in Oberitalien visualisiert ist. ${ }^{32}$ Auch auf einigen Siegeln von Rechtsgelehrten bzw. Medizinern ist so das Bildmotiv des Doctor in Cathedra durch die Abbildung eines oder mehrerer Schüler bzw. Studenten ergänzt, wie beispielsweise auf dem Siegel des Cremoneser Rechtsdoktors Riccardo Malombra, der in Padua kanonisches Recht lehrte, ${ }^{33}$ oder auf dem Siegelstempel des Magisters der Medizin, Pasquzio aus Perugia, der im Museo del Bargello auf-

bor, Michigan, I997 (MikroficheAusgabe) (Dissertation Syracuse University 1996). Zur Typologie von Gelehrtengrabmälern am Beispiel der Grabmäler in der Kirche Sant'Anonio in Padua s. RuTH WolfF, Le tombe dei dottori al Santo: considerazioni sulla loro tipologia, in: Il Santo [2. Ser.] 42.2002 (2003), 277-297.

33 Venedig, Archivio di Stato, Atti diplomatici e privati, b. I I, n. 36I.
Das Consilium ist von E. BestA, Riccardo Malombra, professore nello studio di Padova, consultore di Stato in Venezia, Venezia I 894, 80-82 und Abbildung auf I I I editiert. Zu seinem Inhalt s. BESTA, 54-57. Vgl. auch Il sigillo nella storia e nella cultura, Ausstellung Venezia, Museo Correr, 6.7.3I.8.I98 5 , Katalog hg. v. STEFANIA Ricci, Roma I985, Nr. 97, 99. 
bewahrt und in das I4. Jahrhundert datiert wird. ${ }^{34}$ Das Bildformular des Doctor in Cathedra, der ein aufgeschlagenes Buch in den Händen hält, ist also das Bild eines Gelehrten, der sein Wissen »öffnet « und nach außen mitteilt, wie bei der Unterrichtung des gelehrten Wissens des ius commune an die Studenten innerhalb der Universitäten, aber auch in der Vermittlung und Anwendung dieses Wissens auf Bereiche außerhalb der Universität, wie im Fall der consilia der Rechtsdoktoren. Auf Siegeln taucht das Bildformular weitaus früher als auf Grabmälern auf, was von der bisherigen Forschung noch nicht erkannt wurde. ${ }^{35}$

\section{Die Beziehung von Text und Siegel-Bild}

In seinen Ursprüngen ist das knappe, aber sorgsam konstruierte Bildformular des Doctor in cathedra also eine kongeniale Veranschaulichung des grundsätzlichen Musters des Ratgebens der Rechtsdoktoren - der Vermittlung gelehrten Wissens nach außen -, das sich im Text der Urkunde, an die das Siegel angehängt ist, in einem konkreten Fall (casus) exemplifiziert. Zugespitzt ließe sich sagen: Es ist nicht das Siegel-Bild, das den Text veranschaulicht, sondern der Text illustriert das Bild auf dem Siegel. Diese Art der Text-Bild-Relation trifft auf allgemeine Weise für alle Rechtsgutachten zu, die mit Siegeln mit dem Bild des Doctor in cathedra authentisiert sind. Bild und Text können aber noch spezifischer aufeinander verweisen, wie es der Fall eines weiteren Rechtsgutachtens verdeutlichen soll, dessen Siegel durch die Hinzufügung eines Details zum Bildformular des Doctor in cathedra darüber hinaus auf Vorbilder und Konnotationen dieses Bildformulars verweist. Das consilium des Boncius, decretorum doctor und Prior der Kirche San Martino in Siena, vom I7. August I 3 I 3 ist wie das Gutachten Giovanni d'Andreas in zwei Exemplaren konserviert. ${ }^{36}$ Nur eines der beiden Exemplare (Urkunde A) ist allerdings von einem Notar, hier Meo del fu Riccio, ausgestellt und mit seinem Notariatszeichen versehen. Eine anhängende Siegelschnur verweist auf die ehemalige Besiegelung des Gutachtens. Das andere Exemplar (Urkunde B) ist weder durch einen Notar noch durch ein Siegel authentisiert. Der Abdruck des Siegels von Boncius befindet sich jetzt wie die Siegelabdrücke von Giovanni d'Andreas Rechtsgutachten von I329 in der Sammlung der »sigilli staccati« des Florentiner Staatsarchivs. ${ }^{37} \mathrm{Im}$ Gutachten des Juristen Boncius

34 Florenz, Museo Nazionale del Bargello, Inv. Nr. I 837, Sigilli nel Museo Nazionale, Bd. III, Kat. Nr. 389, I65 f.

35 In der Grabmalsforschung wurde stets das Grabmal des Rolandino dei Passeggeri vor San Domenico in Bologna als erstes Grabmal mit einer Schulszene angesehen (vgl. zuletzt MARIO FANTI, La tomba di Rolandino, monumento dell'ideologia culturale e politica bolognese del Duecento: Giorgio Tamba [Hg.], Rolandino I 2 I 5-I300. Alle origini del notariato moderno, Ausstellung Bologna, Museo Civico Medievale I 2 ottobre-I 7 dicembre 2000, Milano 2002, I IOI I3), das I306 fertig gestellt gewesen sein muss. Zeitgleich ist aber auch in Treviso ein Grabmal mit einer Doctor-in-Cathedra-Szene entstanden, nämlich das Grabmal des Bonincontro de Arpo, doctor legum, das auf dem Friedhof des Doms vor San Giovanni errichtet war und sich jetzt im Depot des Museo di Santa Caterina in Treviso befindet (s. RuTH WOLFF, Zur »Gruppe « der Gelehrtengrabmäler des Mittelalters in Oberitalien, in: Creating identities: die Funktion von Grabmalen und öffentlichen Denkmälern in Gruppenbildungsprozessen, hg. von Wolfgang Neumann, Kassel 2007 [Kasseler Studien zur Sepulkralkultur II], 2 I9-230, hier 222 und Anm. 26, 229). Das Grabmal soll demnächst in einer eigenen Publikation vorgestellt werden.

36 Florenz, Staatsarchiv, Diplomatico Camaldoli, I7.8.13 I3. Das Gutachten ist von AscherI, Analecta consiliare, Nr. 7, 65 f. und Nr. II, $80-83$, ediert.

37 Florenz, Archivio di Stato, im Inventar der » sigilli staccati Nr. 6I. 
geht es um die Gültigkeit einer Urkunde, die eine Belehnung bezeugt. Es ist ebenfalls ein consilium pro parte und wurde bei Boncius von den zwei streitenden Parteien, dem Prior der Eremiten von Camaldoli und einem gewissen Ciapetta von Monte Aguto, angefragt.

Der gut erhaltene Abdruck des Siegels von Boncius ist im Gegensatz zu den Siegelabdrücken am Gutachten Giovanni d'Andreas nicht spitzoval, sondern rund und etwas kleiner (Bild 9). Wieder sehen wir links ein Pult in perspektivischer Verkürzung mit einer doppelten Reihe von schmalen Rundbögen und rechts eine Kathedra und den in ihr thronenden Rechtsgelehrten vor einer

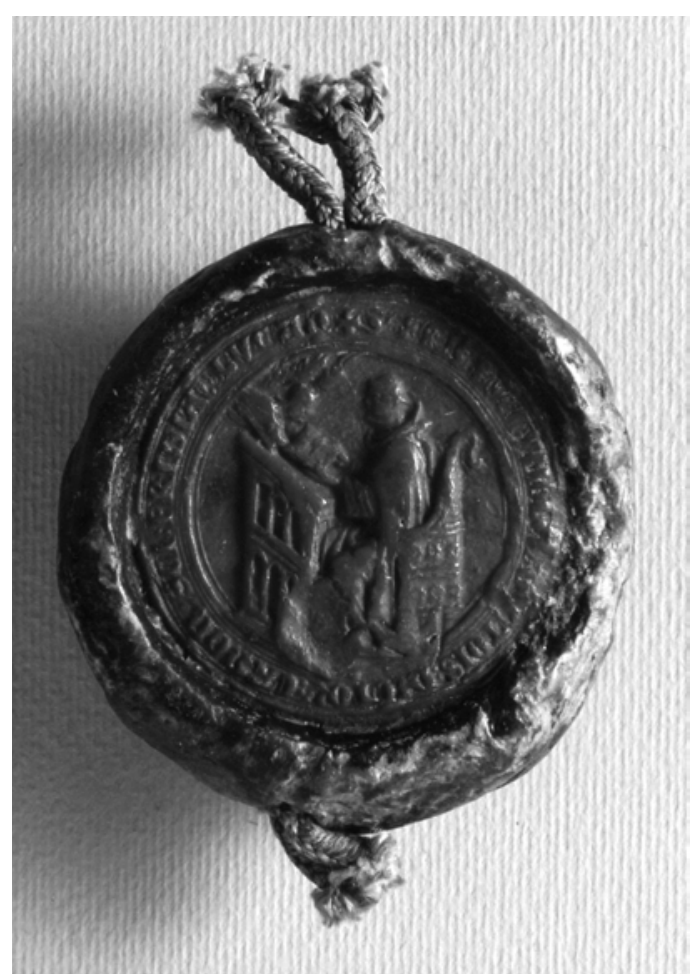

Bild 9: Abdruck des Siegels des Rechtsdoktoren Boncius vom 7.8.I3 33 (Florenz, Archivio di Stato, »sigilli staccati«, Nr. 6I) 
ansonsten leer gelassenen Bildfläche. Auch hier hält der Rechtsdoktor ein aufgeschlagenes Buch in beiden Händen. Seine Bildfigur ist jedoch weniger zentriert positioniert als auf den Siegelabdrücken des Gutachtens Giovanni d'Andreas, und die Kathedra des Rechtsgelehrten und damit auch er selbst sind weniger auf den Betrachter ausgerichtet. Beides erklärt sich aus der Hinzufügung einer Bildfigur, d.h. des Engels, der dem Doktor von links oben herabschwebend das Buch überreicht und ihn ein wenig aus der Bildmitte verdrängt. Die Halbfigur des im Profil dargestellten Engels verlangt zudem die Konzentration des ebenfalls stärker in Seitenansicht abgebildeten Juristen auf den göttlichen Boten.

Das Bild auf dem Siegel von Boncius verweist durch die Hinzufügung des Engels auf eines der Vorbilder der Abbildungen des Doctor in Cathedra auf Siegeln, d. h. auf Darstellungen des göttlich inspirierten Schreibens oder Studierens gelehrter Heiliger oder zuerst der Evangelisten selbst. Das Siegel des Boncius ist das einzige mir bekannte Siegel eines Rechtsdoktoren mit der zusätzlichen Abbildung eines Engels. Es ist jedoch anzunehmen, das dasjenige, was auf dem Siegel des Boncius explizit veranschaulicht ist, also die göttliche, höhere Inspiration des Wissens der Gelehrten, auf Siegel-Bildern ohne die ergänzende Darstellung des Engels durch das beschriebenen Bildformular des Motivs des Doctor in Cathedra implizit mitgemeint ist. Denn im I4. Jahrhundert verändert sich umgekehrt auch die Darstellungsweise gelehrter Heiliger und zuerst des hl. Hieronymus, der jetzt nicht mehr in seiner gestrengen Zelle veranschaulicht wird, sondern in seinem Studiolo, umgeben von Büchern. Bezeichnenderweise war es der Jurist Giovanni d'Andrea, der den Kult des hl. Hieronymus durch sein Liber de Laudibus s. Hieronymi stark förderte. In dem Liber de Laudibus erzählt er, wie er eine besondere malerische Präsentationsweise des Heiligen vorgeschrieben habe: Der hl. Hieronymus solle in der Kathedra thronend gemalt werden, mit dem abgesetzten Hut, der heute von Kardinälen getragen werde, sowie mit dem Löwen. ${ }^{38}$

Die explizite Veranschaulichung der göttlichen Inspiration auf dem Siegel des Doktors des kanonischen Rechts Boncius durch den Engel hat in dem Text des Rechtsgutachtens ihre Entsprechung in der wiederholten und insistierenden Anrufung des göttlichen Beistandes. Wie viele seiner Kollegen und auch Giovanni d'Andrea leitet auch Boncius sein consilium mit der Invokation »In nomine Domini amen« ein. Im Gegensatz zu Giovanni d'Andrea wieder-

38 »Dictavi formam, qua nunc in cathedra sedens pingitur cum capello quo nunc Cardinalis utuntur, deposito, et leonoe mansueto (...) «, zitiert nach Millard Meiss, French and Italian variations on an early fifteenth-century theme: St. Jerome and his study, in: Gazette des beaux-arts 62 (I963) I47-I70, hier I 57. 
39 Ascheri, Analecta consiliare, 82. 40 AsCHERI, Analecta consiliare, 80: In nomine Domini amen. Ego Boncius prior ecclesie sancti Martini Senarum inter decretorum doctores minimus electus et assumptus a venerabili patre domino Accurso priore heremi Camaldulensis (...)

4I Decr.Gr. IX, II, 22,2: Corruit instrumentum, si testes inscripti decesserint, nisi sigillum habeat authenticum, vel a notario sit confectum. Alexander III.: Scripta vero authentica, si testes inscripti decesserint, nisi forte per manum publicam facta fuerint, ita, quod appareant publica, aut authenticum sigillum habuerint, per quod possint probari, non videntur

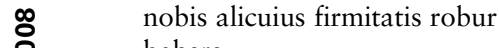
habere.

holt Boncius die Invokation nach der Präsentation des zu beratenden Falls und bevor er seinen Rat abgibt: »Christi nomine invocato, consulendo et declarando dico (...).«39 Die weniger zentrierte Abbildung des Rechtsgelehrten auf seinem Siegel spiegelt sich im Text zudem in der Bescheidenheitsformel wider, mit der sich der Gelehrte einleitend als »inter decretorum doctores minimus « vorstellt und die als Tonfall sein gesamtes Konsilium durchzieht, das sich darin deutlich vom Tenor des Gutachtens Giovanni d'Andreas unterscheidet. ${ }^{40}$

\section{Doppelte Authentisierung}

Das Gutachten des Boncius von I 3 I 3 ist in einem seiner beiden Exemplare und dasjenige des Giovanni d'Andrea von I 329 in beiden Exemplaren von einem Notar geschrieben und besiegelt.

Bereits im Jahr II 8I legte das kanonische Recht unter Berufung auf Papst Alexander III. (II59-II 8I) fest, dass die Beweiskraft einer Urkunde vergehe, wenn ihre Zeugen gestorben seien, es sei denn, sie sei von öffentlicher Hand, d.h. von einem Notar geschrieben oder mit einem authentischen Siegel versehen. ${ }^{4 \mathrm{I}}$ Aus der Sicht der Rechtsgeschichte zeugt die Dekretale Alexanders III. von der frühen Bedeutung des Notariats in Italien, die in vielerlei Hinsicht tatsächlich kaum überschätzt werden kann. ${ }^{42}$ Aus der Sicht der internationalen Siegelkunde hingegen bedeutet die Dekretale eine Aufwertung des Siegels als Authentisierungsmittel. In Italien allerdings habe das Siegel aufgrund der frühen und starken Präsenz des Notariats eine weitaus geringere Rolle als in den anderen europäischen Ländern gespielt. Die Untersuchungen des Projekts "Siegel-Bilder « zeigen ein anderes Ergebnis:

In der Tat ist ein Großteil der in italienischen Archiven aufbewahrten Urkunden von Notaren geschrieben. Viele von ihnen sind tatsächlich nur mittels Unterschrift und signum des Notars authentisiert. Dabei handelt es sich in der Mehrheit um Urkunden, die der Notar im Auftrag und im Interesse von Privatpersonen schrieb, wobei er, wie G. Tamba darstellt, eine übergeordnete Rolle einnahm. Anders im Fall von Notaren, die in kaiserlichen und königlichen Kanzleien oder der päpstlichen Kanzlei tätig waren, für die sie normative, gerichtliche, politische und administrative Urkunden schrieben, die man in weitesten Sinne als »öffentlich" bezeichnen kann: Hier habe der Notar eine diesen »öffentlichen «

42 Zum Notariat in Italien und v. a. in Bologna s. Gianfranco OrLANDELLI, Genesi dell' "ars notarie « nel secolo XIII, in: Studi medievali 3. ser. 6 (I965) 329-368; BRIGIDE SCHWARZ, Das Notariat in Bologna im I3. Jahrhundert, Quellen und Forschungen aus italienischen Archiven und Bibliotheken 53 (I973) 49-92; G. Costamagna, Notaio (diritto intermedio), in: Enciclopedia del diritto, Bd. 28,
Milano I978, 559-565; ANDreas MEYER, "Felix et inclitus notarius «. Studien zum italienischen Notariat vom 7. bis I3. Jahrhundert, Tübingen I986 (Bibliothek des Deutschen Historischen Instituts in Rom 92); Giorgio TAmba, Una corporazione per il potere. Il notariato a Bologna in età comunale, Bologna I998, bes. 7-I 7 I und 299-353. 
Institutionen gegenüber untergeordnete Rolle eingenommen. Notare, die für Kommunen tätig waren, seien dagegen ein Sonderfall, da sie zumeist nur für kurze Zeit berufen wurden, wobei die »öffentliche « Rolle der Kommunen weniger klar definiert gewesen sei und die Notare gleichzeitig für Privatpersonen tätig gewesen seien. ${ }^{43}$

Tamba geht nicht auf die Frage der Besiegelung von Notariatsinstrumenten ein. Seine Beschreibung der über- bzw. untergeordneten Rolle der Notare spiegelt sich jedoch deutlich in den Urkunden und ihrer Besiegelung bzw. Nichtbesiegelung wider: »Privaturkunden ", bei denen der Notar eine untergeordnete Rolle einnahm, bedürfen keiner zusätzlichen Authentisierung durch das Siegel. Urkunden, die vom Notar im Auftrag einer übergeordneten Instanz geschrieben und mit seinem signum und seiner Unterschrift authentisiert werden, werden jedoch zusätzlich mit dem Siegel dieser Instanz authentisiert. Eines von zahlreichen Beispielen einer derartigen doppelten Authentisierung ist eine Urkunde vom 29.IO.I3 I I, in der Bischof Ranieri von Cremona seine Verfügungen von einem Notar » publizieren «, d. h. » in publicam formam « bringen lässt und den Notar beauftragt, das bischöfliche Siegel an der Urkunde anzubringen, ad fidem plenarium adhibendum. ${ }^{44}$

Auch im Gutachten Giovanni d'Andreas und seiner Kollegen vom 9.5.I329 werden in der Subskription der Doktoren die Authentisierung durch das Siegel und den Notar nebeneinander genannt: "So sagen und beraten wir vier Doktoren, indem wir unsere Siegel an dieses Gutachten anbringen lassen und auch den Notar Johannes de Benvenuti de Belvisio beauftragen, aus diesem Gutachten ein öffentliche Urkunde anzufertigen «. ${ }^{45}$ Im Gutachten des Boncius vom I7.8. I 3 I 3 bestätigt der Notar Meus am Ende der Urkunde, dass er beim Vortrag des Gutachtens zugegen gewesen sei, seinen Text im Auftrag des oben genannten dominus Boncius geschrieben, in eine öffentliche Form gebracht und vor den zuvor genannten Zeugen und dem Herrn Prior dessen Siegel mit einer Seidenschnur der Urkunde "eingefügt " habe. ${ }^{46}$ Die beiden in der Dekretale Alexanders III. genannten Möglichkeiten der Authentisierung - durch den Notar und durch das Siegel - wurden in der italienischen Siegelpraxis also nicht als einander ausschließende Alternativen verstanden, sondern häufig simultan und zur wechselseitigen Verstärkung verwendet. Siegel und notarielle Abfassung authentisieren die Rechtsgutachten.

43 ТАмвA, Una corporazione per il potere, I73 f.

44 Perugia, Archivio di Stato, Corporazioni religiose soppresse, San Francesco al Prato, perg. N. 59. Die Verfügung zur publicatio lautet als ganze: »In quorum omnium testimonium predictus venerabilis pater publicari mandavit per Ildibrandinum notarium infrascriptum et, ad fidem plenariam adhibendam, sui sigilli appensione muniri. «Das Siegel der Urkunde ist verloren. Die Urkunde ist publiziert in: Francesco D'Assisi, Documenti e Archivi. Codici e Biblioteche. Miniature, Milano I982, 3 I und Kat. Nr. I7, 35.

45 Ita nos predicti quatuor doctores dicimus et consulimus huic conscilio (sic) nostra facientes apponi sigilla ac etiam mandantes Johanni Benvenuti de Belviso notario ut de ipso consilio publicum conficiat instrumentum.

46 Ego Meus olim Riccii notarius pro lacioni diciti consilii interfui et de mandato supradicti domini Boncii prefatum consilium et suprascripta omnia scripsi et in publicam formam redegi sub anno indictione die et loco et coram testibus suprascriptis et de ipsius domini prioris mandato sigillum suum cum curdella siria huic instrumentum inserui. 
47 Mariano Welber, I sigilli nella storia del diritto medievale italiano, Milano I984 (Bd. III von Sigillograpfia. Il sigillo nella diplomatica, nel diritto, nella storia, nell'arte, Bd. I: Sigillografia generale: i sigilli pubblici e quelli privati, Milano I969, Bd. II: Sigillografia ecclesiastica, Milano I978), hier I $84 \mathrm{ff}$.

48 Zur Verbreitung des Speculum iudiciale im universitären Bereich und seinen zahlreichen Editionen s. Vincenzo Colli, Lo Speculum iudiciale di Guillaume Durand: codice d'autore ed edizione universitaria, in: Juristische Buchproduktion im Mittelalter, hg. v. V. Colli, (Studien zur europäischen Rechtsgeschichte I 55 ), Frankfurt am Main (Klostermann) 2002, 517-566.

49 Guillaume Durant, Speculum iudiciale illustratum et repurgatum a Giovanni Andrea et Baldo degli Ubaldi I 2, Bd. I, Basel I 574 (Neudruck Aalen I975), 623629, bes. 626: »De probationibus: (...) Curia Romana tenet, quod non creditur sigillo, nisi sit au-

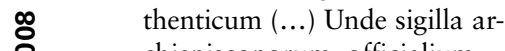
chiepiscoporum, officialium

\section{Authentizität und Autorität}

Das Siegel, das Glauben gegenüber Dritten erzeugt, muss laut der Dekretale Alexanders III. ein authentisches Siegel sein. Mariano Welber, dem wir die einzige Untersuchung zu Siegeln im westlichen Europa und ihrer Stellung im Recht verdanken, die als dritter Band von Giacomo Bascapès "Sigillografia « erschien, betont zu Recht, dass das sigillum authenticum eine Invention des kanonischen Rechts sei, dessen ideologischer Hintergrund das Siegel nicht zufällig und nahezu ausschließlich als Stempel des Göttlichen begreife. ${ }^{47}$ In der Dekretale Alexanders III wird es zum ersten Mal erwähnt, wobei nicht näher bestimmt wird, welche Siegel als authentisch anzusehen seien. Folgerichtig divergieren auch Glossen und Kommentare in der Bestimmung des authentischen Siegels, die u.a. dem jeweils geltenden Gewohnheitsrecht überlassen wird. Auflistungen, welche Siegel als authentisch anzusehen seien, differieren daher voneinander je nach den kulturellen und rechtlichen Voraussetzungen ihres Entstehungsortes. Das Speculum iudiciale des Guillelmus Durantis, das u.a. aufgrund der Schulung seines Verfassers an der Bologneser Universität dem Italien des gelehrten Rechts zugeordnet werden kann und bekanntermaßen von Giovanni d'Andrea und Baldo degli Ubaldi ergänzt wurde ${ }^{48}$ fasst die Problematik des authentischen Siegels folgendermaßen zusammen: »Die römische Kurie ist gehalten, einem Siegel nicht zu glauben, außer wenn es authentisch ist (...) Daher sind die Siegel von Erzbischöfen, ihren Amtsträgern, die von Fürsten sowie von exemten Äbten und die von Notaren, die die Gerichtsbarkeit haben, glaubwürdig in fremden Geschäften, nicht aber die anderer, niederer Äbte, ebenso wenig wie die von Dekanen oder Archidiakonen, es sei denn, diese ständen ihrer Gerichtsbarkeit voran. (...) Die Siegel von Bischöfen sind (...) glaubwürdig, die Siegel von Amtsträgern unterhalb der Bischöfe hingegen nicht (...). Und auch dann genießen sie Glauben, wenn die Gewohnheit so ist, dass ihren Siegeln Glaubwürdigkeit geschenkt wird. Was sie außerhalb des Gerichts bewirken, und inwieweit ihnen geglaubt wird, nämlich nur bis zu einem gewissen Grad, und zwischen welchen Personen ihnen Glaubwürdigkeit zukommt, wird gemäß derjenigen Gewohnheit festgelegt, die die Siegel authentisch macht. « $\mathbf{4 9}$

Giovanni d'Andrea oder Baldo degli Ubaldi glossieren die consuetudo loci in ihren Ergänzungen des Speculum iudiciale und

suorum, et principum, et abbatum exemptorum, et notariorum, iurisdictionem habentium, faciunt fidem in alienis negotiis, non autem aliorum abbatum inferiorum, vel decanorum, vel archidiaconorum, licet praesint iurisdictioni, nisi in his, quae sunt suae iurisdictionis; tunc enim cuilibet ordinario vel delegato creditur (...) Sigilla episcoporum, et supra, faciunt fidem; inferiorum vero epis- coporum non, nisi ut iam dictum est; et nisi consuetudo hoc habeat, quod eorum sigiliis credatur, de his, quae extra iudicium fiunt, et quantum eis credatur, pura usque ad certam summam, et inter quas personas, statur enim in hoc consuetudini, quae facit sigilla authentica (...). "Vgl. auch M. WeLber, Sigilli nella storia del diritto, 22 I f. zur Interpretation des sigillum authenticum durch Durandus 22 I f. 
erläutern, dass beispielsweise in Venedig öffentliche Urkunden (instrumenta) immer noch von Geistlichen (und nicht von Notaren) geschrieben würden. In vielen Gegenden genieße dieses oder jenes Siegel, diese oder jene Unterschrift und dieser oder jener Stempel oder Abdruck Glaubwürdigkeit. Manchmal werde daher eine eigentlich private Urkunde auch als öffentliche Urkunde bezeichnet. Eine solche Urkunde weise nur einen geringen Defekt auf, wenn die Person, die sie geschrieben habe, von großem Ansehen und großer Glaubwürdigkeit (magnae opinionis et fidei), aber kein Notar sei. ${ }^{\circ}$

Hier wird deutlich, wie groß der Spielraum ist, den das Recht der consuetudo zuerkennt: Einerseits sind es bestimmte Formen der Beglaubigung, wie z. B. bestimmte Siegel, die sich in lang anhaltender Tradition durchgesetzt haben, und andererseits ist es die Autorität und Anerkanntheit der Person oder Institution, von der die Urkunde ausgeht, die über die öffentliche Glaubwürdigkeit von Urkunden entscheidet. Auch im Dekretalenrecht wird die Authentizität des Siegels wechselweise auf die honestas des Siegelführers oder den Bekanntheitsgrad und die Integrität des Siegels bezogen. ${ }^{5 \mathrm{I}}$ Giovanni d'Andrea sind die entsprechenden Textstellen gut bekannt und er erläutert sie in seinem um 1338 publizierten Dekretalenkommentar, der Novella Decretalium..$^{52}$

Es kann kein Zweifel daran bestehen, dass Rechtsgelehrte wie Giovanni d'Andrea und viele seiner Kollegen sich selbst als personae magnae opinionis einschätzten und tatsächlich ein ungewöhnlich hohes Sozialprestige genossen. ${ }^{53}$ Nach I. Baumgärtner war es gerade der »intensive Praxisbezug zur Zeit der Kommentatoren oder Konsiliatoren « in der Jurisprudenz, der zu einem » regelrechten Aufstieg eines sich etablierenden Standes « und einer "allmählichen Angleichung an den Adel « geführt habe. Der »nobilitas ex genere «, d.h. dem Geblütsrecht des Adels, sei so neben dem Stand der Geistlichkeit die "nobilitas propter scientiam « der Gelehrten unter Vorherrschaft der Legisten gegenüber gestanden. ${ }^{54}$ Dass ihre Siegel sogar dann als authentische Siegel anerkannt wurden, wenn das Rechtsgutachten nicht von einem Notar geschrieben, sondern nur besiegelt war, zeigt ein Gutachten des Doktors der Rechte Riccardo Malombra aus Cremona von I3 IO/I I. ${ }^{55}$ Es bezieht sich auf Forderungen Kaiser Heinrichs VII. an die Republik Venedig nach zu zahlenden Tributen. Malombra entwickelt und bestätigt in seinem Gutachten die unwiderrufbare Gültigkeit der Stadtgesetz-

50 Speculi carissimi viri Guilelmi Durandi pars secunda ..., I I 7 r, zit. Nach Welber, I93, Anm. I 88: »Unde Venetiis statur instrumentis scriptis per sacerdotes. In multis partibus, facit fidem certum sigillum, certa subscriptio, certa stampa, vel formae impressio. (...) Per praedicta instrumentum privatum dicitur etiam publicum, omnino vero privatum, quod nullum supplementum habet de praedictis. Quandoque patitur defectum modicum, scilicet quando persona scribens est magnae opinionis, et fidei, sed non est tabellio: et habet eius scriptura omnia, quae requiruntur in publico."

5I Zur honestas des Siegelführers s. z. B. die Dekretale Innozenz III. an Bischof von St. Andrews und den Abt von Broch (Dekretalen Capitel 9, X, II, 22): »Super tertio vero articulo taliter respondemus, quod inquiratis diligentius veritatem, et, si consuetudo illius patriae obtinet approbata, ut instrumentis illius regis fides adhibeatur in talibus, vos ea secure poteritis admittere, praesertim quum supradictus rex tantae fuerit honestatis, quod ipsius instrumenta maximae auctoritatis sint in partibus Scoticanis." $\mathrm{Zu}$ den sigilla ignota s. die Dekretale Innozenz III. an den Erzbischof von Canterbury (Dekretalen Capitel VII, X, II, 22).

52 Die relevanten Textstellen finden sich vor allem in Giovannis Kommentar zu De fide instrumentorum und De probationibus, s. IOANNES ANDREAE BonONIENSIS In secundum Decretalium librum Commentaria (quae Novellas appellavit) Acutissima, Venetiis I 58 I (Anast. Neudruck Würzburg I993), De probationibus, I09 ff. und De fide instrumentorum, I 62 ff. Die abschließende Auswertung der Untersuchung von Giovanni d'Andreas Kommentaren und derjenigen seiner Kollegen zu siegelrelevanten Stellen soll in einer größeren Publikation vorgestellt werden. Zur Entstehungszeit und Publikation der Novella s. F. Gillmann, Zur Frage der Abfassungszeit der Novella des Iohannes Andreae, in: Archiv für katholisches Kirchenrecht 104 (I924) 26I-275 und 264-268.

53 Gabriel Le Bras, Velut splendor firmamenti: le docteur dans le droit de l'Eglise médiévale, in: Mélanges Etienne Gilson, Etudes de Philosophie médiévale, Paris I959, 373 ff.; INGRID BAUMGÄRTNER, »De privilegiis doctorum «. Über Gelehrtenstand und Doktorwürde im späten Mittelalter, in: Historisches Jahrbuch, I06 (I986) 298-332 und Giorgio Borelli, »Doctor an miles « : aspetti dell'ideologia nobiliare nell'opera del giurista Cristoforo Lanfranchini, in: Nuova Rivista Storica, anno LXXIII, Gennaio-Aprile I989, I 5 I-I 68.

54 BAUMGäRTNER, "De privilegiis doctorum «, hier besonders 299302.

55 S. Fn. 33. 


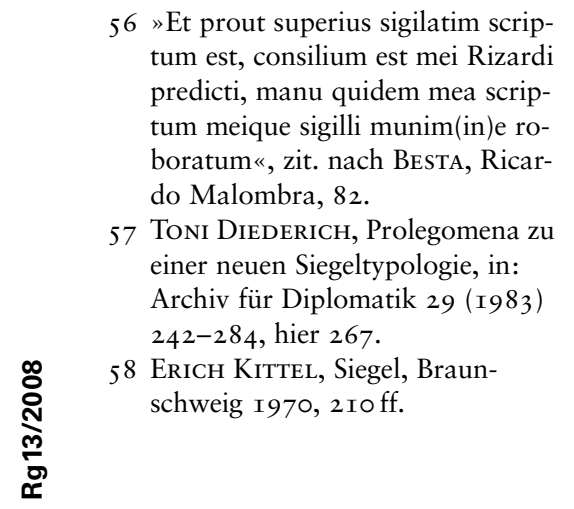

Autorität und Authentizität

gebung Venedigs auch gegenüber einer höheren Autorität wie der kaiserlichen. An das consilium ist der bereits erwähnte spitzovale Wachsabdruck seines Siegels angehängt mit dem Motiv des Doctor in Cathedra in dem hier analysierten Bildformular, das durch zwei in kleinerem Figurenmaßstab abgebildete Studenten ergänzt ist. Wie aus der Korrobationsformel hervorgeht, ist das consilium nicht von einem Notar geschrieben, sondern von Malombra selbst und allein mit dem Siegel Malombras bekräftigt. ${ }^{56}$

Sowohl der Anspruch der Siegel von Rechtsgelehrten auf Authentizität als auch ihre Rezeption als sigilla authentica treten jedoch am deutlichsten anhand des Siegel-Bildes zu Tage. Denn die überragende Mehrheit der Siegel von Rechtsgelehrten veranschaulichen das Motiv des Doctor in Cathedra und damit ein Bild des Siegelführers selbst. Siegel mit dem Bild des Siegelführers werden in der Sphragistik als »Repräsentationsmittel von hohem Rang « eingestuft, ${ }^{57}$ das nur hochrangigen Personen vorbehalten war. So präsentieren sich Könige und Kaiser seit Kaiser Otto III. und den Jahren $997 / 98$ bis zu Kaiser Karl V. mit dem Bild des frontal thronenden Herrschers. ${ }^{58}$ Der Herrscherstand repräsentierte sich dagegen mit dem Bild des Reiters auf dem Pferd, in Frankreich bereits seit dem II. Jahrhundert, mit einer Blütezeit im I3. bis zum Ausklang im 16. Jahrhundert. ${ }^{59}$ Bischöfe haben sich wie Gelehrte nicht nur, doch vorwiegend mit einem Bild ihrer selbst auf Siegeln veranschaulichen lassen. M. Groten hat z. B. das »Aufkommen der bischöflichen Thronsiegel im Deutschen Reich « untersucht, die sich in der ersten Hälfte des I 2. Jahrhunderts gegenüber dem Brustbild der Bischöfe aus politischen Motiven durchsetzten. ${ }^{60}$ In anderen Ländern, wie Frankreich und England, spielte das Thronbildsiegel bei Bischöfen keine Rolle, sondern hier war das Bild des stehenden und segnenden Bischofs dominant, ${ }^{6 \mathbf{I}}$ ebenso wohl auch in Italien, über dessen Bischofssiegel keine eigenständigen Untersuchungen vorliegen.

Rechtsgelehrte stellen sich mit ihrem Siegel-Bild also auf gleiche Ebene mit weltlichen und geistlichen Herrschern. Dies gelingt nur, weil sich viele Rechtsgelehrte verschiedener Orte und über einen langen Zeitraum diesem gemeinsamen Siegel-Bild unterordnen, und zwar nicht nur hinsichtlich des Bildmotivs, sondern vor allem in Bezug auf das Bildformular, das sich als ein sehr sorgfältig konstruiertes erwiesen hat. Seine Stärke liegt darin, dass es sich nicht auf die immergleiche Wiedergabe von »Attributen«

59 Kittel, Siegel, 250 ff.

60 Manfred Groten, Das Aufkommen der bischöflichen Bischofssiegel im deutschen Reich, in: Historisches Jahrbuch roo (1980) I63-197. Zu französischen Bischofssiegeln s. RoBerTHenri Bautier, Apparition, diffusion et évolution typologique du sceau épiscopale au Moyen Age, in: Die Diplomatik der Bischofsurkunde vor I250: La diploma- tique épiscopale avant I250; Referate zum VIII. Internationalen Kongress für Diplomatik, Innsbruck, 27. September-3. Oktober I993, hg. von Christoph HaIDACHER Und WERNER KÖFLER, Innsbruck I995, 269-280.

6I W. H. ST. John Hope, Seals of English Bishops, in: Proceedings of the Society of Antiquaries of London, 2nd. Ser. II, 27I-306. 
wie Kathedra, Pult und Buch beschränkt, sondern den Rechtsgelehrten in einer Körperhaltung visualisiert, die sein Bild auf Siegeln auf den ersten Blick von Bildern anderer hochrangiger Siegelführer unterscheidbar macht, also von dem Bild des frontalen Thronens der Kaiser und Könige oder des hieratischen Stehens der Bischöfe. Trotz des sehr kleinen Bildformats wird die Wiedererkennbarkeit nicht durch eine abstrahierende und summarische, sondern vielmehr durch eine verfeinerte Bildsprache erreicht, wobei die Plastizität von Siegelstempel und Siegelabdruck, das Hervortreten und Zurückliegen einzelner Bildpartien und der Wechsel von Licht und Schatten eine bedeutende Rolle spielt. Denn erst durch die gesteigert formulierte räumliche Darstellung des Gelehrten in leichter Körperdrehung werden einzelne Bildelemente stärker hervorgehoben, andere mehr zurückgesetzt, und das Bildformular kann auch aus größerem Abstand klarer als eigenständiger Bildtypus wahrgenommen werden. Dadurch und durch die insistierende Wiederholung des Bildtypus auf Siegeln vieler Rechtsgelehrter werden diese zu sigilla nota, denen Glaubwürdigkeit geschenkt wird.

Siegel von Rechtsgelehrten erweisen sich so als anschauliches Beispiel für die Genese eines authentischen Siegel-Bildes, das sich durch ortsübergreifende und lang anhaltende consuetudo zu behaupten vermag und in engem Zusammenhang mit den consilia der Rechtsgelehrten entsteht.

Ruth Wolff 